\title{
Image De-Noising and Enhancement - A Comparative Study on Ultrasound TOFD Scans
}

\author{
Srinivasan R \\ SASTRA University, \\ Thanjavur, India
}

\author{
Ramamoorthi P V \\ SASTRA University, \\ Thanjavur, India
}

\author{
Vijayarekha K \\ SASTRA University, \\ Thanjavur, India
}

\author{
Venkataraman B \\ Indira Gandhi Centre for \\ Atomic Research, \\ Kalpakkam, India
}

\begin{abstract}
Non-destructive Evaluation (NDE) has become one of the most widely used technique for flaw detection due to its many advantages. One major problem faced in the process is the pre-processing of the obtained b-scan image to eliminate the system-generated noise. Not many processing techniques exist for the de-noising of such images given the random nature of the noise present. This paper gives an account of some of the de-noising and enhancement techniques which can be used for Time of Flight Diffraction (TOFD) technique for detecting weld defects. Results of the application of these techniques to the actual images obtained from scanning are shown, and the techniques are also compared with the aim of finding the most suitable one. In this paper, we have utilized various filters and transforms and have estimated the results by calculating the Peak Signal-Noise Ratio (PSNR) of each of the processed images. The final processed images have been tabulated and presented for different real-time scan signals.
\end{abstract}

\section{General Terms}

Time of Flight Diffraction (TOFD), Non-Destructive Evaluation (NDE), Weld Defects, Image enhancement

\section{Keywords}

De-noising, Image Enhancement, Filter, Curvelets, Wavelets, TOFD

\section{INTRODUCTION}

In order to evaluate the strength and residual life of welded components, it is necessary to non-destructively characterize the weld defect. For this, the ideal detection tool has been found to be the TOFD technique, which overcomes the limitations of the previously existing techniques like X-ray imaging on various counts. Even in the case of near-surface flaws $(<3 \mathrm{~mm}$ depth), Ultrasound TOFD technique has been found to be very accurate. The results of these techniques can be obtained in various forms as required for the task at hand, unlike the X-ray, which gives only one type of output irrespective of the necessity. The most common modes of presenting the scans are the A-scan (displays the amount of received ultrasonic energy as a function of time), B-scan (profile or cross-sectional view of the test specimen) and Cscan (plan-type view of the location and size of test specimen features). In this paper, we have used the B-scan images of welded joints and have presented various methods to process it.
A major problem faced with the ultrasound scanning technique is the noise which is added to the scan image. The noise is of random nature, hence making the de-noising procedure furthermore difficult. Also, due to the influence of imaging mode, acoustic characteristics of the material under test and the testing system, characteristic information of the defect is often obscured in the raw TOFD scan image. Hence, it is necessary to enhance the image in order to clearly define the scan characteristics and extract the useful information from the raw data.A large number of de-noising techniques are in existence, but not all can be used to process the ultrasound images. This is due to the fact that the scan is to be treated as an information representation rather than an ordinary image. In this paper, we present some of the techniques - both de-noising and enhancement, which can be used on these images and give a comparative analysis of the same. These techniques are necessary as traditional threshold based de-noising techniques are often rendered useless, given that the gray scale variations in the scan are very small. The pre-processing of the image will mainly aim at manipulating the contrast of the image in order to highlight the features of the defect.

\section{IMAGE ACQUISITION}

TOFD experiment model Microplux of M9S AEA technology, U.K with manual scanner along with longitudinal wave (4 MHz) angle beam probe of 450 (model WSY 45) was used for the experiment. A manual weld scanner was used in acquisition of the B-scans obtained by scanning the welded bead. These scan results were further analyzed for flaw detection using powerful Image Processing tools in MATLAB.

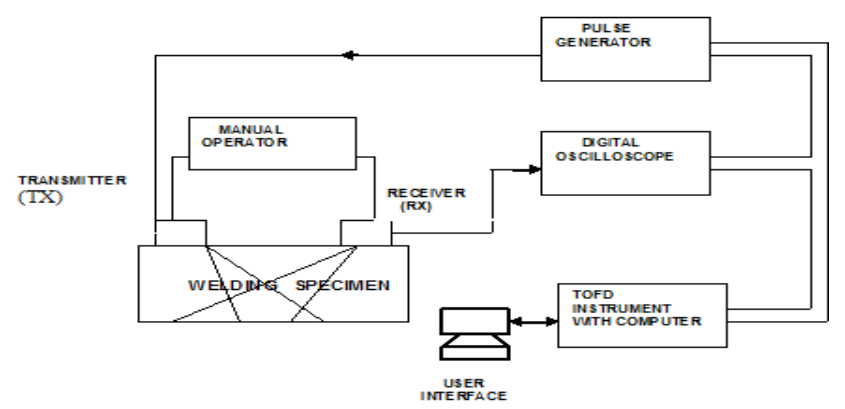

Figure 1: Experimental setup for getting TOFD welding defect signals. 


\section{IMAGE DENOISING}

\subsection{Linear Filter in the Spatial Domain:}

A low-pass filter can be used on a gray-scale image that has been degraded by constant power additive noise. We have used an adaptive Wiener Filter ${ }^{[1]}$ which uses a pixel-wise adaptive Wiener method ${ }^{[2]}$ based on statistics estimated from a local neighborhood of each pixel.

First, the local mean and variance around each pixel are estimated:

$\mu=\frac{1}{N M} \sum_{n 1, n 2 \in \eta} x(n 1, n 2)$

$\sigma^{2}=\frac{1}{N M} \sum_{n 1, n 2 \in \eta} x^{2}(n 1, n 2)-\mu^{2}$

$\eta$ is the N-by-M local neighborhood of each pixel in the image $\mathrm{X}$. Then a pixel-wise wiener filter can be defined using these estimates:

$y(n 1, n 2)=\mu+\frac{\sigma^{2}-v^{2}}{\sigma^{2}}(x(n 1, n 2)-\mu)$

Here, $v^{2}$ is the noise variance. This approach often produces better results than ordinary linear filtering. The adaptive filter is more selective than a comparable linear filter, preserving edges and other high-frequency parts of an image.

\subsection{2-D Mean and Median Filters:}

The median filter is somewhat similar to the mean filter, only it does a better job of preserving useful detail in the image. The median filter is also a sliding-window spatial filter, but it replaces the center value in the window with the median of all the pixel values in the window instead of the mean, as in the case of the mean filter. Median filters can do an excellent job of rejecting certain types of noise, in particular, "shot" or impulse noise in which some individual pixels have extreme values.

\begin{tabular}{|l|l|l|}
\hline 43 & 14 & 16 \\
\hline 13 & 2 & 22 \\
\hline 23 & 76 & 35 \\
\hline
\end{tabular}

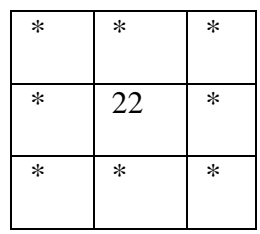

Figure 2: Unfiltered Data (left) and values of the block after performing median filtering

Median filtering does not shift boundaries, as can happen with conventional smoothing filters (a contrast dependent problem). Also, since the median is less sensitive than the mean to extreme values (outliers), those extreme values are more effectively removed. The median is, in a sense, a more robust "average" than the mean, as it is not affected by outliers.

\subsection{DWT De-noising}

As defined by the mathematician Gilbert Strang, a wavelet, in the sense of a Discrete Wavelet Transform (DWT) ${ }^{[3][4]}$, is an orthogonal function which can be applied to a finite group of data. It is quite similar to the Discrete Fourier Transform - the transforming function is orthogonal, a signal passed twice through the transformation is unchanged and the input signal is assumed to be a set discrete-time samples. The discrete wavelet transform (DWT) is an implementation of the wavelet transform using a discrete set of the wavelet scales and translations obeying some defined rules. The wavelet can be constructed from a scaling function which describes its scaling properties:

$$
\phi(x)=\sum_{k=-\infty}^{\infty} a_{k} \phi(S x-k) .
$$

where $S$ is a scaling factor (usually chosen as 2). The area between the function must be normalized and scaling function must be orthogonal to its integer translates. The wavelet is obtained from the scaling function as:

$$
\psi(x)=\sum_{k=-\infty}^{\infty}(-1)^{k} a_{N-1-k} \psi(2 x-k)
$$

Here $\mathrm{N}$ is an even integer.

Wavelet de-noising attempts to remove the noise present in the signal while preserving the signal characteristics, regardless of its frequency content and involves three steps - a linear forward wavelet transform, non-linear thresholding and a linear inverse wavelet transform. By applying DWT, the image is actually divided i.e., decomposed into four sub-bands and critically sub sampled. The sub bands labeled LH1, HL1 and $\mathrm{HH} 1$ represent the finest scale wavelet coefficients, i.e., detail images while the sub band LL1 corresponds to coarse level coefficients, i.e., approximation image. To obtain the next coarse level of wavelet coefficients, the sub band LL1 alone is further decomposed and critically sampled. This results in two-level wavelet decomposition.

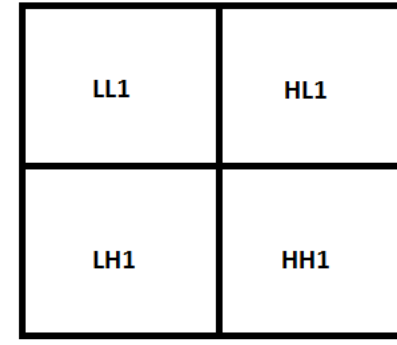

One-level

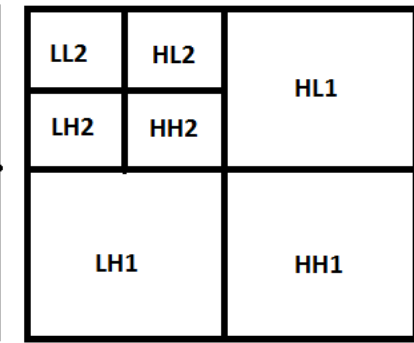

Two-level
Figure 3: Wavelet decomposition representation 
Wavelet de-noising method relies on the fact that noise commonly manifests itself as fine-grained structure in the image and DWT provides a scale based decomposition. Thus, most of the noise tends to be represented by wavelet coefficients at the finer scales. Discarding these coefficients would result in a natural filtering of the noise on the basis of scale.

The threshold plays an important role in the de-noising process. Finding an optimum threshold is a tedious process. A small threshold value will retain the noisy coefficients whereas a large threshold value leads to the loss of coefficients that carry image signal details. Normally, hard thresholding and soft thresholding techniques are used for such de-noising process. Hard thresholding is a keep or kill

rule whereas soft thresholding shrinks the coefficients above the threshold in absolute value (shrink or kill rule).

\subsection{ROF De-noising:}

The total variation (TV) ${ }^{[5]}$ based image de-noising model of Rudin, Osher, and Fatemi ${ }^{[6]}$ can be generalized in a natural way to privilege certain edge directions. Given an observed

intensity function $f$ they reconstruct the clean image $u$ assuming $f=u+\eta$, where $\eta$ is an additive noise. They propose to minimize the following function:

$$
J(u)=\int_{\Omega}|\nabla u|+\lambda \int_{\Omega}(f-u)^{2}
$$

for a certain tuning parameter $\lambda>0$. The set $\Omega$ is a domain of $\mathrm{R}^{\mathrm{n}}$ and the term $\int_{\Omega}\left|\nabla_{\mathrm{u}}\right|$ denotes the total variation of $\mathrm{u}$, assuming $\mathrm{u}$ is of bounded variation: $\mathrm{u} \in \mathrm{BV}(\Omega)$. The above equation is called the Rudin-Osher-Fatemi Algorithm (ROF).

The total variation of the image is minimized subject to constraints involving the statistics of the noise. The constraints are imposed using Lagrange multipliers. The solution is obtained using the gradient-projection method. This amounts to solving a time dependent partial differential equation on a manifold determined by the constraints. The method is noninvasive, yielding sharp edges in the image. This is a two-phase process where the noise and textures are first isolated by scalar TV. The adaptive process then imposes local power constraints based on local variance measures of the first phase.

\section{IMAGE ENHANCEMENT}

\subsection{Homomorphic Filter:}

The homomorphic filter removes the low frequency variations due to illumination by taking the log of the image before high pass filtering and then the exponent to display the result. Homomorphic filtering can perform simultaneous dynamic range compression and contrast enhancement. The selection of an appropriate frequency-domain filter function is crucial for the success of the homomorphic approach in order to modify the illumination and reflectance components of an image differently. To make the illumination of an image more even, the high-frequency components are increased and lowfrequency components are decreased, because the highfrequency components are assumed to represent mostly the reflectance in the scene (the amount of light reflected off the object in the scene), whereas the low-frequency components are assumed to represent mostly the illumination in the scene. That is, high-pass filtering is used to suppress low frequencies and amplify high frequencies, in the log-intensity domain.

We have used a Butterworth filter together with $\log / \exp$ to perform homomorphic filtering.

\subsection{Contrast Limited Adaptive Histogram Equalization (CLAHE):}

In CLAHE, the contrast limiting procedure has to be applied for each neighborhood [7] from which a transformation function is derived. CLAHE operates on smaller portions of the image called tiles instead of the whole image itself. The artificially introduced boundaries are then eliminated using bilinear interpolation technique. CLAHE has the advantage of prevention of over-amplification of the noise signal, when compared to ordinary enhancement techniques.

\subsection{The Curvelet Transform:}

Curvelets are band-limited complex-valued functions $\Phi_{\alpha \beta \theta}$ : R2 $\rightarrow \mathrm{C}$ parameterized in a scale $(\alpha>0) /$ location $(\beta \in \mathrm{R} 2) /$ rotation $(\theta \in \mathrm{S} 1)$ space. The graph of the modulus of a curvelet looks like a brush stroke of a given thickness (as given by $\alpha>0)$, location on the canvas $(\beta \in \mathrm{R} 2)$, and direction $(\theta \in \mathrm{S} 1)$.

Curvelet Analysis ${ }^{[8][9]}$ is the right tool to locate artifacts with a linear structure: The high scale curvelets code information of curvilinear features such as path singularities, and also any other structure that can be well approximated by ellipses with one axis considerably longer than the other.By gathering curvelet coefficients of the same scaling level, one obtains partial reconstructions of the original image where directional features of different thicknesses are enhanced. Linear combinations of these partial reconstructions are considered as approximations of the enhanced image. The advantage of using curvelets comes from the fact that the order of decay of the coefficients $\left\{\left\langle f, \Phi_{\alpha \beta \theta}\right\rangle\right\}_{\alpha}$ gives extra information about the type of singularity.

\section{RESULTS AND DISCUSSION}

The enhanced and de-noised images of a sample are shown below: 


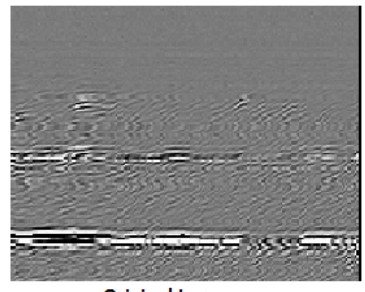

Original Image

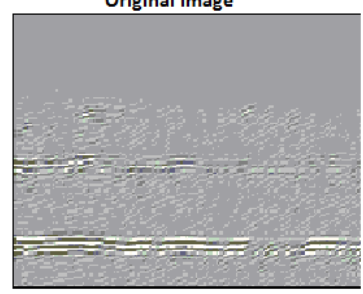

Median Filtered

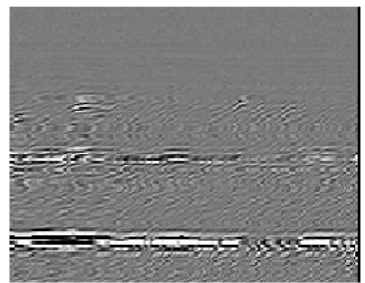

Original Image

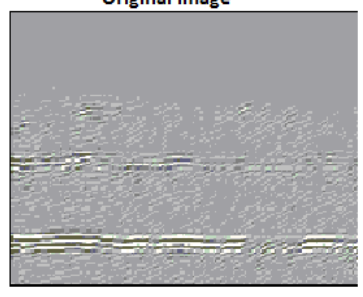

Median Filtered

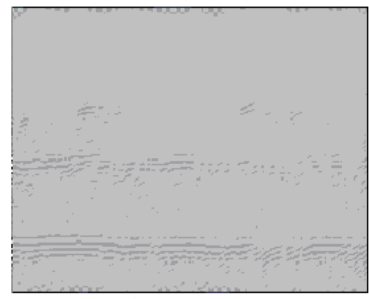

Homomorphic Filtered

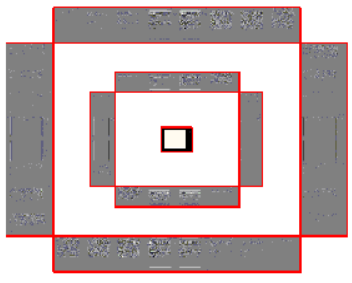

Curvelet Transform applied to original image

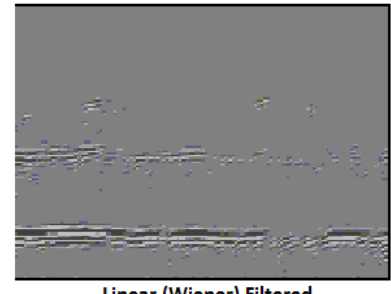

Linear (Wiener) Filtered

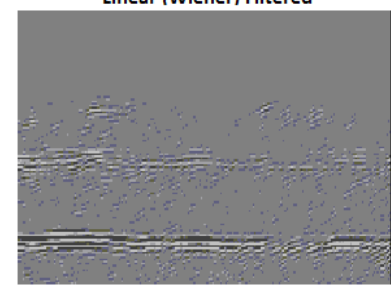

DWT De-noised

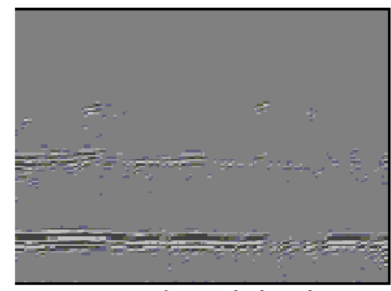

Linear (Wiener) Filtered

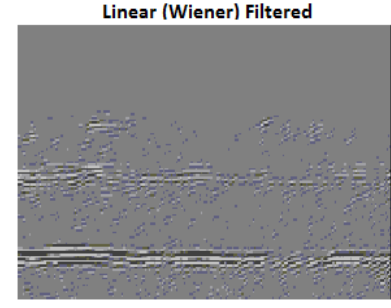

DWT De-noised

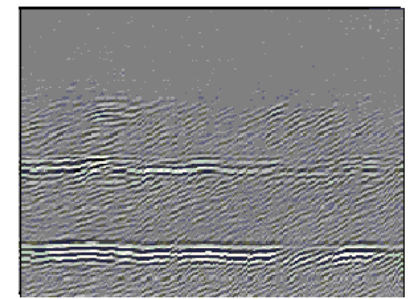

CLAHE Enhanced
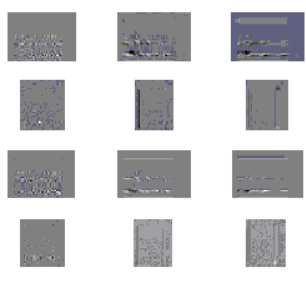

Curvelet Coefficients

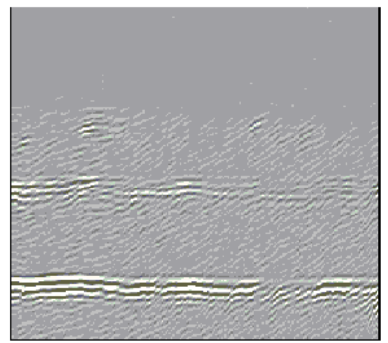

Curvelet Transform Enhanced

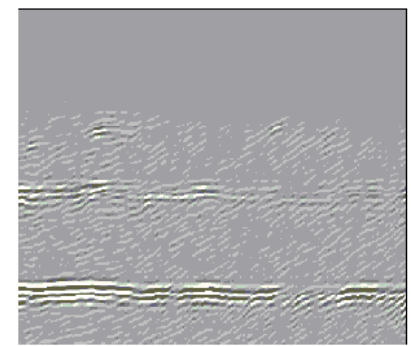

ROF Enhanced
The PSNR values of the processed images are calculated and tabulated as shown.

\begin{tabular}{|l||l||l|l||}
\hline Process & Scan1 & Scan2 & Scan3 \\
\hline \hline Linear Filter & 31.644 & 35.453 & 37.697 \\
\hline Median Filter & 30.814 & 34.330 & 36.756 \\
\hline DWT de-noising & 31.173 & 33.423 & 35.675 \\
\hline Homomorphic Filter & 27.393 & 28.654 & 30.974 \\
\hline CLAHE & 30.506 & 30.974 & 32.084 \\
\hline Curvelet Enhancement & 32.959 & 36.876 & 38.098 \\
\hline ROF Enhancement & 31.573 & 34.052 & 36.341 \\
\hline \hline
\end{tabular}

\section{INFERENCES AND CONCLUSIONS}

From the above table, it is observed that the Curvelet and the ROF Enhancement techniques a better PSNR compared to the rest of the enhancement/de-noising technique. In recovering images which are smooth away from edges, curvelets will obtain dramatically smaller asymptotic mean square error of reconstruction than wavelet methods. The high PSNR value is indicative of the fact that the Curvelet enhancement algorithms better suited for the pre-processing of TOFD signals compared to the others. The ROF enhancement and DWT de-noising are close behind, suggesting that improvements in the parameters of the algorithm may yield better results.

\section{FUTURE WORK}

Image de- noising is a necessary step in many image processing applications. Hence, a good algorithm needs to be selected for a particular type of application based on parameters like PSNR, efficiency atc. Neural network based classifiers can be used to select the optimum algorithm for a particular application.

\section{ACKNOWLEDGMENTS}

Our sincere thanks to IGCAR, Kalpakkam for providing us with the data, without which this paper would not have been possible. We would also like to thank SASTRA University for the invaluable support provided to us in publishing this paper. 


\section{REFERENCES}

[1] S. Mallat, 'Wavelet Tour of Signal Processing', Academic Press (1998)

[2] G. Chang, B Yu and M. Vetterli, 'Spatially adaptive wavelet thresholding with context modeling for image denoising', preprint (1998)

[3] Sachin D Ruikar and Dharmpal D Doye, 'Wavelet Based Image Denoising Technique', (IJACSA) International Journal of Advanced Computer Science and Applications,Vol. 2, No.3, March 2011

[4] R. Sivakumar, K. Gayathri and D. Nedumaran, 'Speckle Filtering of Ultrasound B-Scan Images - A Comparative Study of Single Scale Spatial Adaptive Filters, Multiscale Filter and Diffusion Filters', IACSIT International Journal of Engineering and Technology, Vol.2, No.6, December 2010, ISSN: 1793-8236

[5] Y. Yu and S. T. Acton, 'Edge detection in ultrasound imagery using the instantaneous coefficient of variation', IEEE Trans. Med. Imag., vol. 13, no. 12, pp. 1640-1655, 2004.
[6] Rudin, L. I.; Osher, S. J.; Fatemi, E. 'Nonlinear total variation based noise removal algorithms', Physica D (1992), 259 - 268.

[7] Virendra P. Vishwakarma, Sujata Pandey and M. N. Gupta, 'Adaptive Histogram Equalization and LogarithmTransform with Rescaled Low Frequency DCTCoefficients for Illumination Normalization', International Journal of Recent Trends in Engineering Vol 1, No. 1, May 2009

[8] Jean-Luc Starck, Emmanuel J. Candès, and David L. Donoho, 'The Curvelet Transform for Image Denoising', IEEE Transactions On Image Processing, Vol. 11, No. 6, June 2002

[9] Shadi AlZubi, Naveed Islam and Maysam Abbod, 'Multiresolution Analysis Using Wavelet, Ridgelet, and Curvelet Transforms for Medical Image Segmentation', International Journal of Biomedical Imaging Volume 2011 (2011), Article ID 136034, 18 pages 